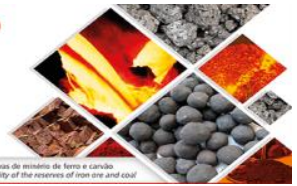

\title{
ESTUDOS DE CORRELAÇÃO DE CONCENTRAÇÃO MAGNÉTICA NAS ESCALAS DE BANCADA, PILOTO E INDUSTRIAL*
}

\author{
Nilson Nedes de Paula \\ George Eduardo Sales Valadão ${ }^{2}$ \\ Antônio Eduardo Clark Peres ${ }^{3}$
}

\section{Resumo}

Este trabalho visa apresentar os resultados de um estudo de correlação envolvendo concentração magnética nas escalas bancada-piloto e piloto-industrial. Os testes que sustentam este estudo foram realizados no CPT (Centro de Pesquisas Tecnológicas da Vale). A correlação entre as escalas piloto-industrial tende a ser direta, uma vez que, concentradores magnéticos piloto possuem os mesmos mecanismos de atuação de um industrial. Por outro lado, entende-se que a correlação entre as escalas bancada-piloto não seja tão direta assim, necessitando de um entendimento/conhecimento maior para descrevê-la. Aproveitou-se este trabalho também para estudar uma forma de melhorar o modelo atual utilizado pelo CPT em interpretações mineralógicas voltadas para concentração magnética. A metodologia deste trabalho foi dividida em seis etapas distintas: escolha das amostras, amostragem industrial, caracterização das amostras, interpretação mineralógica, testes piloto, testes de bancada e análise de correlação. Em relação aos resultados, este trabalho confirma a interpretação mineralógica como uma boa ferramenta para estimar recuperação mássica e qualidade de concentrado em processos de concentração magnética, e que modelo utilizado atualmente pelo CPT apresenta melhores resultados em relação à modificação proposta neste trabalho. Os testes piloto apresentam resultados praticamente idênticos aos resultados da industrial, confirmando que a correlação entre as escalas piloto-industrial é direta. Por fim, este trabalho confirma que é possível correlacionar os resultados de bancada com os resultados da piloto. Os gráficos de correlação apresentam $\mathrm{R}^{2}$ superior a 0.77 , podendo se basear na utilização de equações de regressão para estimar resultados piloto, a partir dos resultados de bancada. Entretanto, entende-se que é necessário realizar teste complementares, uma vez que, o espaço amostral (número de amostras estudadas) foi pequeno.

Palavras chave: Concentração magnética; Correlação; Testes piloto; Testes bancada.

\section{CORRELATION ANALYSIS OF MAGNETIC CONCENTRATION IN LABORATORY, INDUSTRIAL AND PILOT SCALES}

\begin{abstract}
This paper presents the results of correlation study involving magnetic concentration in pilot-industrial and laboratory-pilot scales. The tests that support this study were carried out in TRC ( Vale Company Technology Research Center). The correlation between the pilot and industrial scale tends to be straightforward, once that pilot magnetic concentrators are similar industrial magnetic concentrators. Moreover, it's understood that the correlation between laboratory and pilot scales is not as straightforward. They require a greater understanding/knowledge to describe them. Also took up this work to study a way improving the current model used by TRC in mineralogical interpretations about magnetic concentration process. The study methodology was divided in six phase: choice of samples, industrial sampling, characterization of the samples, mineralogical interpretation, pilot test, laboratory test and correlation analysis. Regarding the results, this study confirms the mineralogical interpretation as a good way to estimate mass recovery and concentrate quality in magnetic concentration process. Moreover and model currently used by TRC presents better results compared to modifications proposed this paper. The pilot tests results are similar to industrial results, confirming the correlation between pilot and industrial scales is straightforward. Finally, this study confirms it's possible to correlate the laboratory and pilot results, using regression equations to estimate pilot results from laboratory results, once that the graphs presents good correlation. However, it's understood that on moment for this work is not be possible, since the sample space is small.
\end{abstract}

Keywords: Magnetic concentration; Correlation; Pilot test; Laboratory test.

1 Engenheiro de Processo, Centro de Pesquisas Tecnológicas, Vale, Brasil. Mestrando do Programa de PósGraduação em Engenharia Metalúrgica e de Minas da Universidade Federal de Minas Gerais, Belo Horizonte, MG, Brasil.

2 Professor do Departamento de Engenharia de Minas da Universidade Federal de Minas Gerais, Belo Horizonte, MG, Brasil.

3 Professor do Departamento de Engenharia Metalúrgica da Universidade Federal de Minas Gerais, Belo Horizonte, MG, Brasil.

* Contribuição técnica ao 44ํ Seminário de Redução de Minério de Ferro e Matérias-primas, 15은 Simpósio Brasileiro de Minério de Ferro e 2o Simpósio Brasileiro de Aglomeração de Minério de Ferro, 15 a 18 de setembro de 2014, Belo Horizonte, MG, Brasil. 


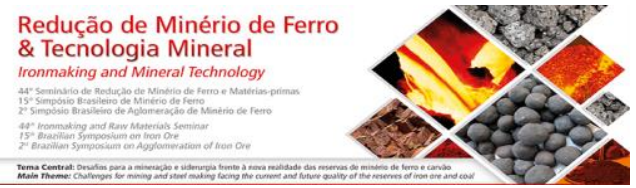

\section{INTRODUÇÃO}

\subsection{Apresentação}

A indústria de minério de ferro dos tempos atuais tem cada vez mais investido em estudos de caracterização tecnológica envolvendo o processo de concentração magnética. Isso se deve em muito a uma boa eficiência desse processo frente aos minérios atuais, associado a uma maior exigência do mercado em relação à qualidade dos produtos de minério de ferro. A caracterização tecnológica de minérios é ferramenta importante no aproveitamento de um recurso mineral de forma otimizada, sendo recomendada nas fases de exploração e explotação de um bem mineral. De modo geral, uma estrutura de caracterização tecnológica, empregada na definição e/ou melhoria de circuitos de concentração magnética, engloba testes em escala de laboratório, piloto e industrial, tendo como suporte análises física, química e mineralógica. Este trabalho visa apresentar os resultados de um estudo de correlação envolvendo concentração magnética nas escalas bancada-piloto e pilotoindustrial. Os testes que sustentam este estudo foram realizados no CPT (Centro de Pesquisas Tecnológicas da Vale. Aproveitou-se também este trabalho para estudar uma forma de melhorar o modelo atual utilizado pelo CPT em interpretações mineralógicas voltadas para concentração magnética. $O$ teste piloto de concentração magnética consome uma quantidade muito grande de amostra, além de necessitar de um número maior de pessoas para sua realização. Dessa forma se torna muito difícil a realização de testes piloto com amostras de furo de sonda. $\mathrm{Na}$ caracterização tecnológica de minério de ferro envolvendo concentração magnética, geralmente os resultados dos testes de bancada não são utilizados de forma direta nos projetos, pelo fato de se conhecer muito pouco sobre o scale up destes com os resultados de testes contínuos, o que acaba colocando em necessidade a realização de testes piloto. Utilizar correlações para prever resultados piloto a partir de resultados de bancada pode trazer ganhos aos trabalhos de caracterização envolvendo concentração magnética. Para realizar um teste piloto de concentração magnética, atualmente no CPT é necessário uma massa de amostra de aproximadamente $80 \mathrm{~kg}$. Caso sejam realizados somente testes de bancada, essa massa reduziria para $20 \mathrm{~kg}$, ou seja, quatro vezes menos. Uma redução significativa, que proporcionaria ganhos tangíveis como: redução de custos (amostra de furo de sonda possui custo elevado) e redução de tempo (testes piloto por serem mais complexos, são também mais morosos). Assim, surge-se a necessidade de estudar e buscar um melhor entendimento sobre as correlações existentes entre as escalas: bancada, piloto e industrial.

\subsection{Objetivos}

Estabelecer para concentradores magnéticos tipo WHIMS (wet high intensity magnetc separator), a correlação existente entre resultados das escalas: bancadapiloto e piloto-industrial. Propor uma revisão do modelo atual utilizado pelo Centro de Pesquisas Tecnológicas da Vale, para estimar recuperação mássica e qualidade de concentrado através de interpretação mineralógica voltada para concentração magnética.

\footnotetext{
* Contribuição técnica ao 44 Seminário de Redução de Minério de Ferro e Matérias-primas, 15ㅇ Simpósio Brasileiro de Minério de Ferro e $2^{\circ}$ Simpósio Brasileiro de Aglomeração de Minério de Ferro, 15 a 18 de setembro de 2014, Belo Horizonte, MG, Brasil.
} 


\subsection{Revisão da Literatura}

- Origem das Propriedades Magnéticas dos Minerais: as propriedades físicas dos minerais são a expressão macroscópica da sua constituição interna, especialmente de sua estrutura cristalina e composição química. Os minerais podem ser separados quando submetidos a campos magnéticos de alta intensidade por apresentarem uma ampla gama de valores para a susceptibilidade magnética. Os minerais denominados diamagnéticos não são atraídos por um campo magnético. Alguns minerais conhecidos como paramagnéticos podem ser atraídos por um campo magnético. Os minerais fortemente magnéticos são conhecidos como ferromagnéticos [1]. Estes, além de apresentarem os átomos característicos da família dos elementos de transição, são caracterizados por todos os dipolos magnéticos individuais apresentarem a mesma direção quando submetidos a um campo magnético. Esse fenômeno ocorre devido à sobreposição de orbitais de átomos vizinhos na rede cristalina dos minerais. A tabela 1 apresenta os íons mais comuns dos elementos de transição, sua configuração eletrônica e momento magnético expresso em termos do magnéton de Bohr [2].

Tabela 1: Composição Íons mais comuns dos elementos de transição, sua configuração eletrônica e momento magnético expresso em termos do magnéton de Bohr.

\begin{tabular}{|c|c|c|c|}
\hline Íons & Configuração & $\begin{array}{c}\text { Elétrons desemparelhados no } \\
\text { orbital d }\end{array}$ & Momento magnético \\
\hline $\mathrm{Ti}^{3+}, \mathrm{V}^{4+}$ & $\hat{1}$ & 1 & $1 \mu_{\beta}$ \\
\hline $\mathrm{Ti}^{2+}, \mathrm{V}^{3+}$ & $\hat{1} \hat{\imath}$ & 2 & $2 \mu_{\beta}$ \\
\hline $\mathrm{V}^{2+}, \mathrm{Cr}^{3+}, \mathrm{Mn}^{4+}$ & $\hat{\imath} \hat{\imath} \hat{\imath}$ & 3 & $3 \mu_{\beta}$ \\
\hline $\mathrm{Cr}^{2+}, \mathrm{Mn}^{3+}$ & $\hat{1} \hat{1} \hat{1} \hat{1}$ & 4 & $4 \mu_{\beta}$ \\
\hline $\mathrm{Mn}^{2+}, \mathrm{Fe}^{3+}$ & $\hat{1} \hat{1} \hat{1} \hat{1} \hat{1}$ & 5 & $5 \mu_{\beta}$ \\
\hline $\mathrm{Fe}^{2+}, \mathrm{Co}^{3+}$ & $\hat{\imath} \hat{\imath} \hat{\imath} \hat{I} \hat{I}$ & 6 & $6 \mu_{\beta}$ \\
\hline $\mathrm{Co}^{2+}$ & $\hat{\imath} \hat{\imath} \hat{\imath} \hat{\imath} \hat{1}$ & 7 & $7 \mu_{\beta}$ \\
\hline $\mathrm{Ni}^{2+}$ & $\hat{\imath} \hat{I} \hat{\perp} \hat{\imath} \hat{I}$ & 8 & $8 \mu_{\beta}$ \\
\hline $\mathrm{Cu}^{2+}$ & $\hat{\imath} \mathfrak{A} \downarrow \hat{I} \downarrow \hat{I} \downarrow \hat{I}$ & 9 & $9 \mu_{\beta}$ \\
\hline $\mathrm{Zn}^{2+}, \mathrm{Cu}^{+}$ & $\hat{\imath} \downarrow \hat{\imath} \downarrow \hat{\imath} \downarrow \hat{\imath} \downarrow \hat{\imath}$ & 10 & 0 \\
\hline
\end{tabular}

- Concentradores Magnéticos de alta intensidade (WHIMS): a separação ocorre nos pacotes magnéticos localizados na circunferência de dois rotores equipados com certo número de placas ranhuradas equidistantes umas das outras conforme apresentado na figura 1 . As placas podem apresentar diferentes tipos de ranhuras, as configurações mais comuns são com 4, 8 e 12 ranhuras por polegadas ao longo do comprimento da placa (linear). A capacidade dos separadores é função do volume de polpa que pode passar pelos pacotes magnéticos, que por sua vez é função da distância entre as placas e da largura da matriz [3].

* Contribuição técnica ao 44ํㅗㄴ Seminário de Redução de Minério de Ferro e Matérias-primas, $15^{\circ}$ Simpósio Brasileiro de Minério de Ferro e 2ํ Simpósio Brasileiro de Aglomeração de Minério de Ferro, 15 a 18 de setembro de 2014, Belo Horizonte, MG, Brasil. 

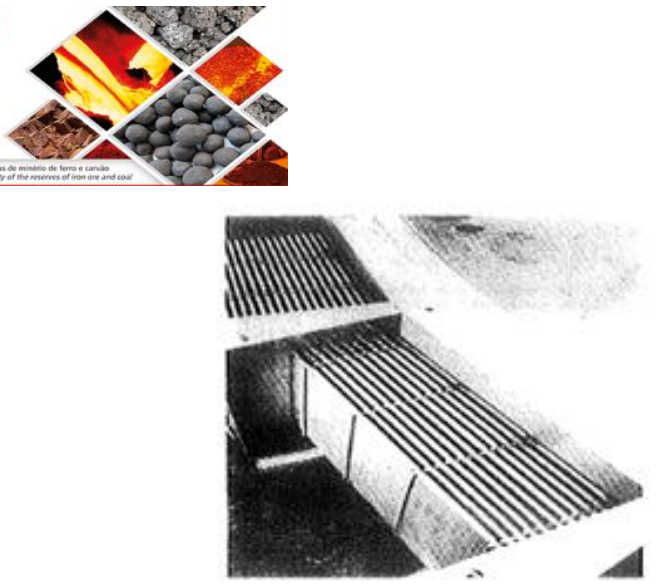

Figura 1: llustração dos pacotes magnéticos localizados na circunferência de dois rotores equipados com certo número de placas ranhuradas equidistantes umas das outras.

- Concentrador Eletromagnétco de Bancada Modelo L4: fabricado pela empresa INBRAZ, possui como mecanismo físico de concentração uma matriz de separação fixa entre dois polos magnéticos induzidos. É operado por batelada e utilizado com boa eficiência na estimativa de qualidade para concentração magnética de média e alta intensidade de campo magnético. $\mathrm{O}$ máximo da intensidade de campo magnético depende do gap em utilização. Por exemplo: para gap de 2,5 mm e 1,5 mm, o campo máximo é de 9000 Gauss e 13000 Gauss, respectivamente. A figura 2 apresenta fotos ilustrativas do L4 [4].
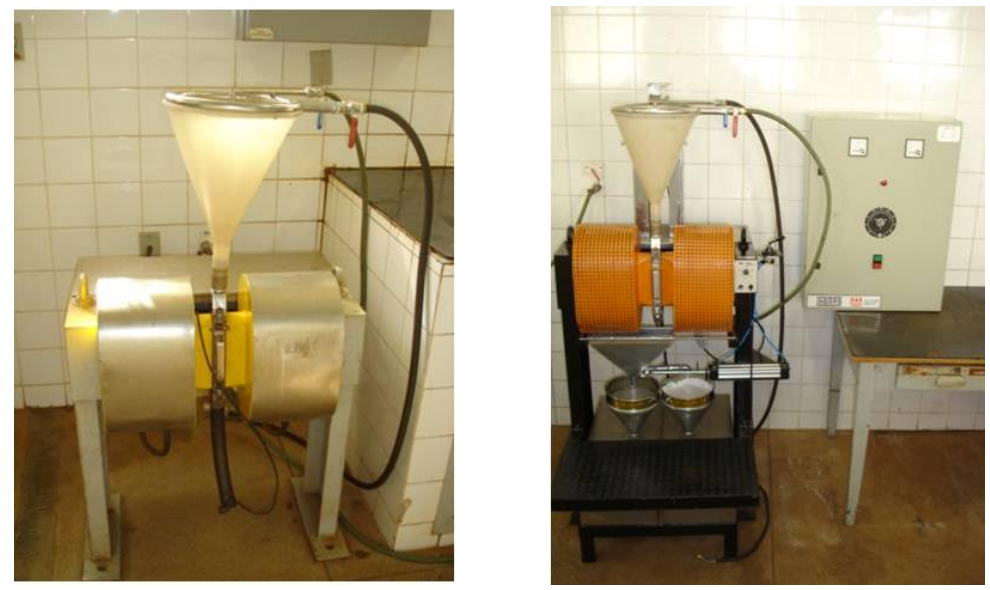

Figura 2: Fotos ilustrativas do concentrador eletromagnético de bancada modelo L4.

- Concentrado Eletromagnético Piloto Modelo Minimag: fabricado pela empresa GAUSTEC, é um concentrador WHIMS de carrossel em escala piloto, via úmido de alta intensidade de campo magnético (máximo de 18000 Gauss com gap de $1,5 \mathrm{~mm}$ ). As partículas magnéticas são atraídas pelo campo eletromagnético e ficam aderidas às placas de imantação (constituindo concentrado), as não magnéticas são descartadas por arraste hidráulico e pela gravidade (constituindo rejeito) e as partículas mistas são descartadas por ação entre forças competitivas (constituindo médio). A figura 3 apresenta fotos ilustrativas do Minimag [4].

* Contribuição técnica ao 44ํㅗㄴ Seminário de Redução de Minério de Ferro e Matérias-primas, $15^{\circ}$ Simpósio Brasileiro de Minério de Ferro e 2ํ Simpósio Brasileiro de Aglomeração de Minério de Ferro, 15 a 18 de setembro de 2014, Belo Horizonte, MG, Brasil. 

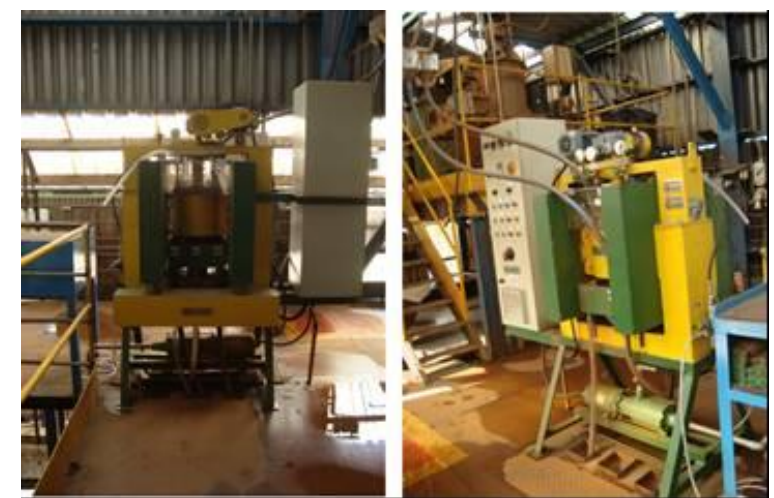

Figura 3: Fotos ilustrativas do concentrador eletromagnético piloto modelo Minimag.

\section{MATERIAIS E MÉTODOS}

Este trabalho foi realizado em seis etapas distintas: escolha das amostras, amostragem industrial, caracterização das amostras, interpretação mineralógica com estimativa de recuperação mássica e qualidade para o concentrado magnético, testes de concentração magnética em escala piloto/bancada e análise de correlação entre as escalas supracitadas. Todos os testes, a preparação de amostra, as granulometrias e as análises/interpretações mineralógicas foram realizadas no CPT. As análises química foram realizadas por fluorescência de Raio-X no laboratório químico da Vale. No primeiro momento foram escolhidas cinco amostras que representam alimentação do WHIMS na industrial: rejeito da flotação de Alegria, underflow da deslamagem de Cauê, undersize do peneiramento de alta frequência de Brucutu (alimentação do Jones rougher de Brucutu), alimentação do Jones cleaner de Brucutu e underflow do circuito de classificação da ITM-I do Pico (alimentação do Jones rougher do Pico). Visando ratificar os resultados alcançados na primeira batelada de testes, no segundo momento, foram escolhidas mais cinco amostras para realização de testes adicionais. Essas amostras já se encontravam arquivada no CPT. O modelo atualmente utilizado pelo CPT para estimar recuperação mássica e qualidade de concentrado magnético, é realizado a partir da fração global de uma determinada amostra. Visando melhorá-lo, foi realizado na etapa de interpretação mineralógica, a classificação das amostras nas frações granulométricas: $>0,045 \mathrm{~mm}$ e $<0,045 \mathrm{~mm}$. O objetivo disso é comparar as duas formas, correlacionando as estimativas mineralógicas com os resultados dos ensaios de bancada. A figura 4 apresenta o fluxograma simplificado das principais atividades realizadas neste trabalho.

* Contribuição técnica ao 44ำ Seminário de Redução de Minério de Ferro e Matérias-primas, 15은 Simpósio Brasileiro de Minério de Ferro e 2ํ Simpósio Brasileiro de Aglomeração de Minério de Ferro, 15 a 18 de setembro de 2014, Belo Horizonte, MG, Brasil. 


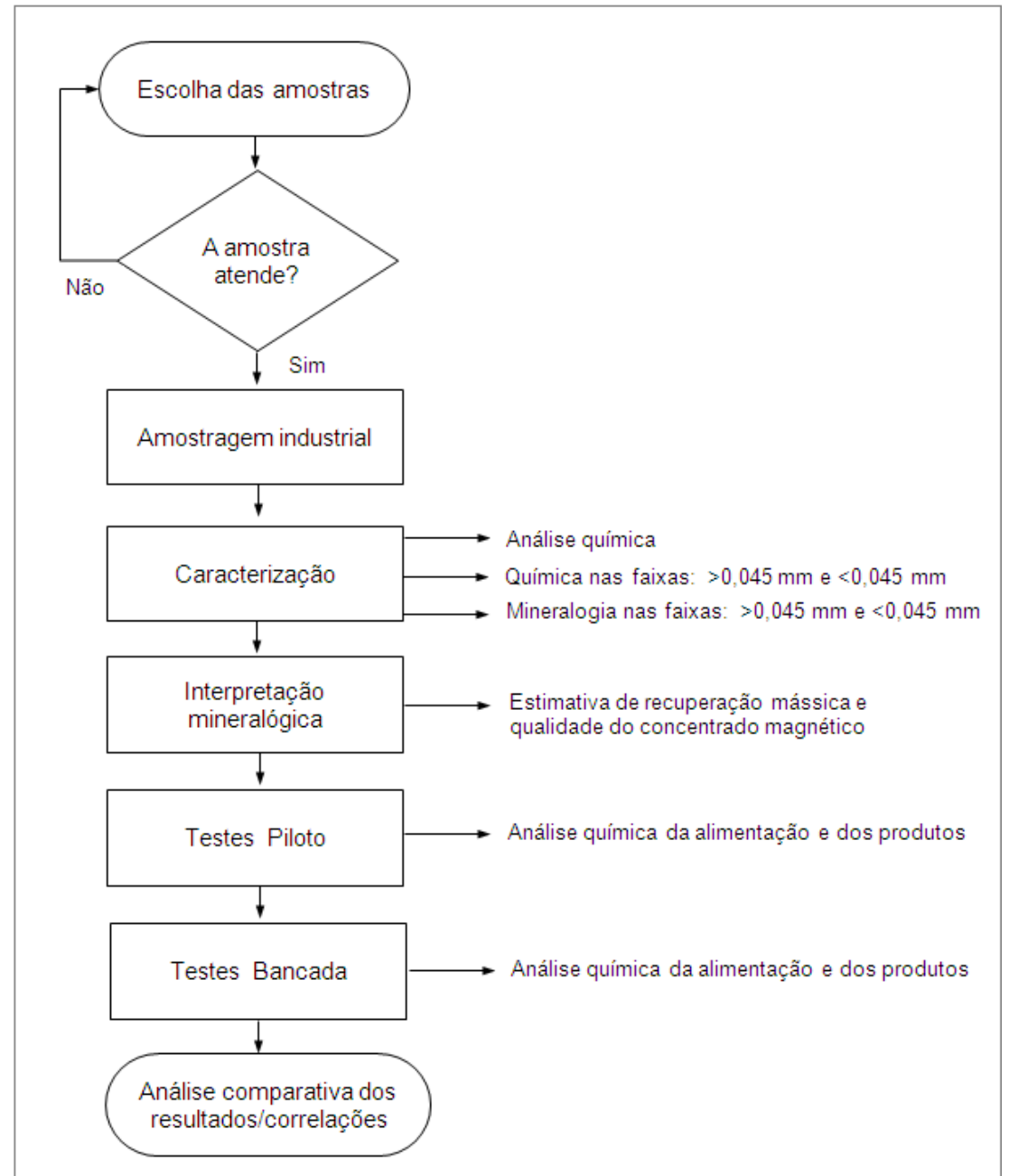

Figura 4: Fluxograma simplificado da metodologia do trabalho.

\section{RESULTADOS E DISCUSSÃO}

\subsection{Interpretação Mineralógica}

As figuras 5 e 6 apresentam os gráficos de correlação da estimativa mineralógica com os resultados obtidos nos testes de bancada propriamente ditos, sendo que na figura 7 os valores estimados são referentes às análises com as amostras fracionadas nas faixas $>0,045 \mathrm{~mm}$ e $<0,045 \mathrm{~mm}$. Entende-se que o espaço amostral é pequeno, em função da base de dados possuir somente cinco amostras. De qualquer forma, percebe-se que as correlações encontradas são boas, indicando a interpretação mineralógica como uma boa ferramenta a ser utilizada nos trabalhos de caracterização tecnológica, uma vez que, consegue prever para o processo de concentração magnética, o potencial de concentrabilidade de uma determinada amostra. Isso tende a proporcionar benefício como: a redução do número de testes de bancada a serem realizados e o tempo de caracterização gasto em um determinado estudo. Verifica-se ainda que a condição de amostra global apresenta correlações mais fortes que a amostra fracionada, sugerindo maior assertividade do modelo utilizado atualmente pelo CPT.

* Contribuição técnica ao 44 Seminário de Redução de Minério de Ferro e Matérias-primas, $15^{\circ}$ Simpósio Brasileiro de Minério de Ferro e 2ํ Simpósio Brasileiro de Aglomeração de Minério de Ferro, 15 a 18 de setembro de 2014, Belo Horizonte, MG, Brasil. 

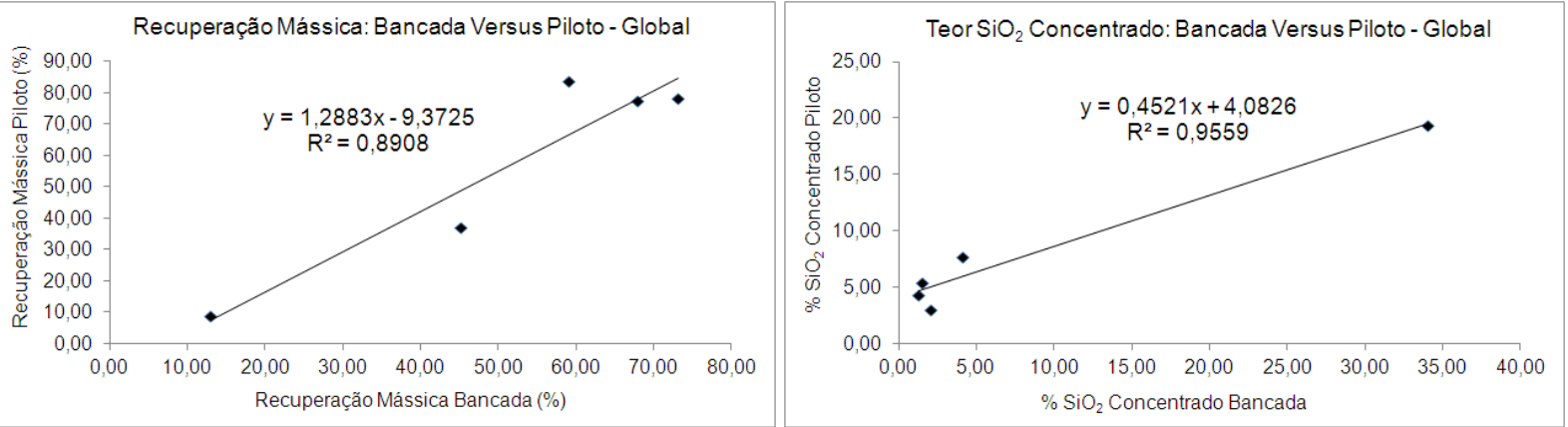

Figura 5: Estimativa mineralógica versus resultados bancada - Amostra Global.
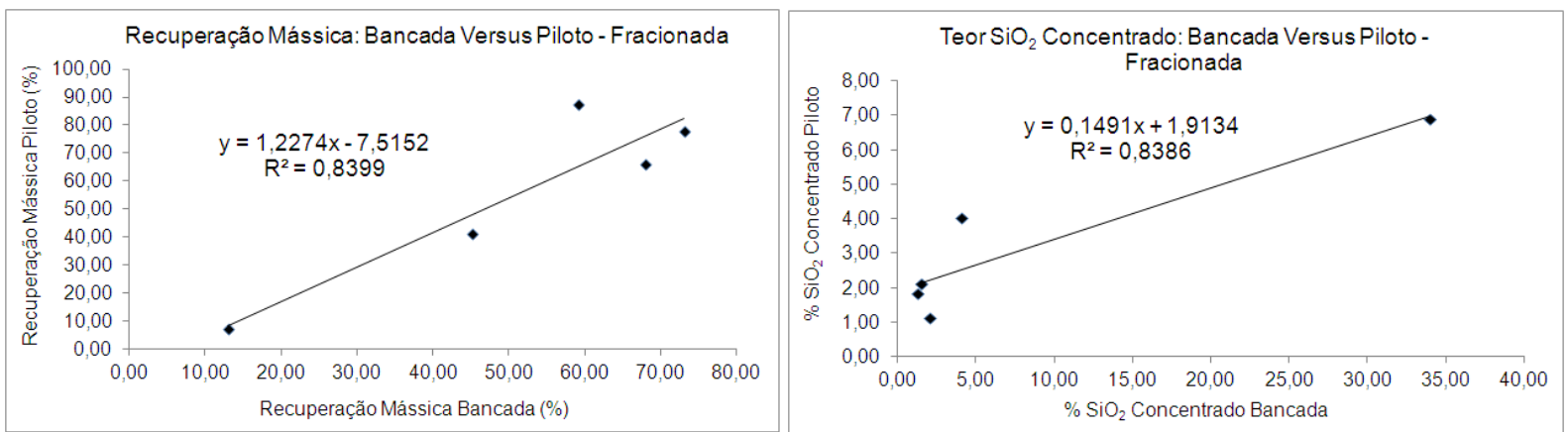

Figura 6: Estimativa mineralógica versus resultados bancada - Amostra Fracionada.

\subsection{Testes Piloto}

A tabela 2 apresenta os resultados dos testes piloto realizados na primeira parte deste trabalho. Todos foram executados no concentrador eletromagnético "Minimag". Os parâmetros utilizados foram os mesmos da industrial. Ressalta-se que os testes piloto complementares realizados na segunda parte deste trabalho serão apresentados juntamente com os resultados dos testes de bancada. Visando estabelecer parâmetros de comparação, paralelamente são apresentados os resultados da industrial. A figuras 7 apresenta gráficos de correlação para: recuperação mássica, teor de $\mathrm{Fe}$ e $\mathrm{SiO}_{2}$ no concentrado e teor de $\mathrm{Fe}$ no rejeito. Verifica-se para todas as amostras que os resultados da piloto são praticamente idênticos aos resultados da industrial, apresentando correlações fortes com $R^{2}$ acima de 0,97. Assim, entende-se que não é necessário aplicar nenhum fator de conversão dos resultados piloto para a industrial. Eles podem ser utilizados diretamente nos projetos de minério de ferro, no que tange a configuração e dimensionamento de circuitos de concentração magnética.

* Contribuição técnica ao 44ํㅗㄴ Seminário de Redução de Minério de Ferro e Matérias-primas, 15은 Simpósio Brasileiro de Minério de Ferro e 2ํ Simpósio Brasileiro de Aglomeração de Minério de Ferro, 15 a 18 de setembro de 2014, Belo Horizonte, MG, Brasil. 
Tabela 2 - Resultados dos testes piloto versus industrial.

\begin{tabular}{|c|c|c|c|c|c|c|c|c|c|c|c|}
\hline \multirow{2}{*}{ Amostra } & \multirow{2}{*}{ Escala } & \multirow{2}{*}{ Fluxo } & \multirow{2}{*}{$\begin{array}{l}\text { Recuperaçãoo } \\
\text { Mássica (\%) }\end{array}$} & \multirow{2}{*}{$\%$ Sólidos } & \multicolumn{5}{|c|}{ Análise Química Global (\%) } & \multirow{2}{*}{$\begin{array}{l}\text { Campo } \\
\text { (Gauss) }\end{array}$} & \multirow{2}{*}{$\begin{array}{l}\text { Gap } \\
\text { (mm) }\end{array}$} \\
\hline & & & & & $\mathrm{Fe}$ & $\mathrm{SiO}_{2}$ & $P$ & $\mathrm{Al}_{2} \mathrm{O}_{3}$ & Mn & & \\
\hline \multirow{6}{*}{$\begin{array}{c}\text { Alimentação } \\
\text { Jones Finos } \\
\text { Cauê }\end{array}$} & \multirow{3}{*}{ Industrial } & Alimentação & 100,00 & 55,00 & 40,91 & 40,38 & 0,015 & 0,715 & 0,075 & \multirow{3}{*}{9.000} & \multirow{3}{*}{2,5} \\
\hline & & Concentrado & 63,20 & - & 61,00 & 11,48 & 0,018 & 0,559 & 0,086 & & \\
\hline & & Rejeito & 36,80 & - & 6,39 & 90,02 & 0,009 & 0,981 & 0,056 & & \\
\hline & \multirow{3}{*}{ Piloto } & Alimentação & 100,00 & 52,42 & 40,05 & 41,11 & 0,019 & 0,797 & 0,087 & \multirow{3}{*}{9.000} & \multirow{3}{*}{2,5} \\
\hline & & Concentrado & 61,75 & - & 60,96 & 11,10 & 0,019 & 0,518 & 0,079 & & \\
\hline & & Rejeito & 38,25 & - & 6,28 & 89,51 & 0,018 & 1,220 & 0,099 & & \\
\hline \multirow{6}{*}{$\begin{array}{c}\text { Alimentação } \\
\text { Jones } \\
\text { Alegria }\end{array}$} & \multirow{3}{*}{ Industrial } & Alimentação & 100,00 & 32,22 & 9,54 & 84,69 & 0,008 & 0,875 & 0,005 & \multirow{3}{*}{13.000} & \multirow{3}{*}{1,5} \\
\hline & & Concentrado & 16,00 & - & 40,51 & 39,46 & 0,022 & 1,107 & 0,001 & & \\
\hline & & Rejeito & 84,00 & - & 3,64 & 93,30 & 0,006 & 0,831 & 0,006 & & \\
\hline & \multirow{3}{*}{ Piloto } & Alimentação & 100,00 & 30,29 & 9,39 & 85,24 & 0,010 & 0,758 & 0,001 & \multirow{3}{*}{13.000} & \multirow{3}{*}{1,5} \\
\hline & & Concentrado & 12,65 & - & 46,16 & 32,47 & 0,015 & 0,855 & 0,003 & & \\
\hline & & Rejeito & 87,35 & - & 4,06 & 92,88 & 0,009 & 0,744 & 0,001 & & \\
\hline \multirow{6}{*}{$\begin{array}{c}\text { Alimentação } \\
\text { Jones } \\
\text { Rougher } \\
\text { Brucutu }\end{array}$} & \multirow{3}{*}{ Industrial } & Alimentação & 100,00 & 35,00 & 57,44 & 16,12 & 0,009 & 0,476 & 0,012 & \multirow{3}{*}{12.600} & \multirow{3}{*}{2,5} \\
\hline & & Concentrado & 87,70 & - & 64,24 & 6,40 & 0,010 & 0,451 & 0,013 & & \\
\hline & & Rejeito & 12,30 & - & 8,99 & 85,43 & 0,007 & 0,650 & 0,008 & & \\
\hline & \multirow{3}{*}{ Piloto } & Alimentação & 100,00 & 35,05 & 56,64 & 17,81 & 0,010 & 0,415 & 0,011 & & \\
\hline & & Concentrado & 86,96 & - & 63,49 & 8,17 & 0,009 & 0,368 & 0,010 & 12.600 & 2,5 \\
\hline & & Rejeito & 13,04 & - & 10,98 & 82,04 & 0,014 & 0,728 & 0,015 & & \\
\hline & & Alimentação & 100,00 & 40,00 & 61,70 & 9,96 & 0,009 & 0,463 & 0,010 & & \\
\hline & Industrial & Concentrado & 91,93 & - & 64,80 & 5,59 & 0,009 & 0,436 & 0,010 & 9.000 & 2,5 \\
\hline Jones & & Rejeito & 8,07 & - & 26,39 & 59,76 & 0,013 & 0,771 & 0,009 & & \\
\hline Cleaner & & Alimentação & 100,00 & 40,33 & 61,28 & 10,76 & 0,017 & 0,540 & 0,009 & & \\
\hline & Piloto & Concentrado & 89,94 & - & 65,18 & 5,39 & 0,017 & 0,420 & 0,010 & 9.000 & 2,5 \\
\hline & & Rejeito & 10,06 & - & 24,79 & 61,44 & 0,027 & 1,124 & 0,013 & & \\
\hline & & Alimentação & 100,00 & 30,00 & 57,25 & 13,80 & 0,059 & 1,042 & 0,360 & & \\
\hline & Industrial & Concentrado & 66,56 & - & 63,85 & 5,14 & 0,052 & 0,758 & 0,254 & 12.000 & 3,8 \\
\hline Jones & & Rejeito & 33,44 & & 44,12 & 31,02 & 0,072 & 1,605 & 0,571 & & \\
\hline Rougher & & Alimentação & 100,00 & 29,39 & 56,98 & 15,14 & 0,044 & 0,850 & 0,275 & & \\
\hline & Piloto & Concentrado & 60,67 & - & 66,23 & 3,08 & 0,033 & 0,420 & 0,126 & 12.000 & 3,8 \\
\hline & & Rejeito & 39,33 & - & 42,68 & 33,83 & 0,064 & 1,667 & 0,563 & & \\
\hline
\end{tabular}
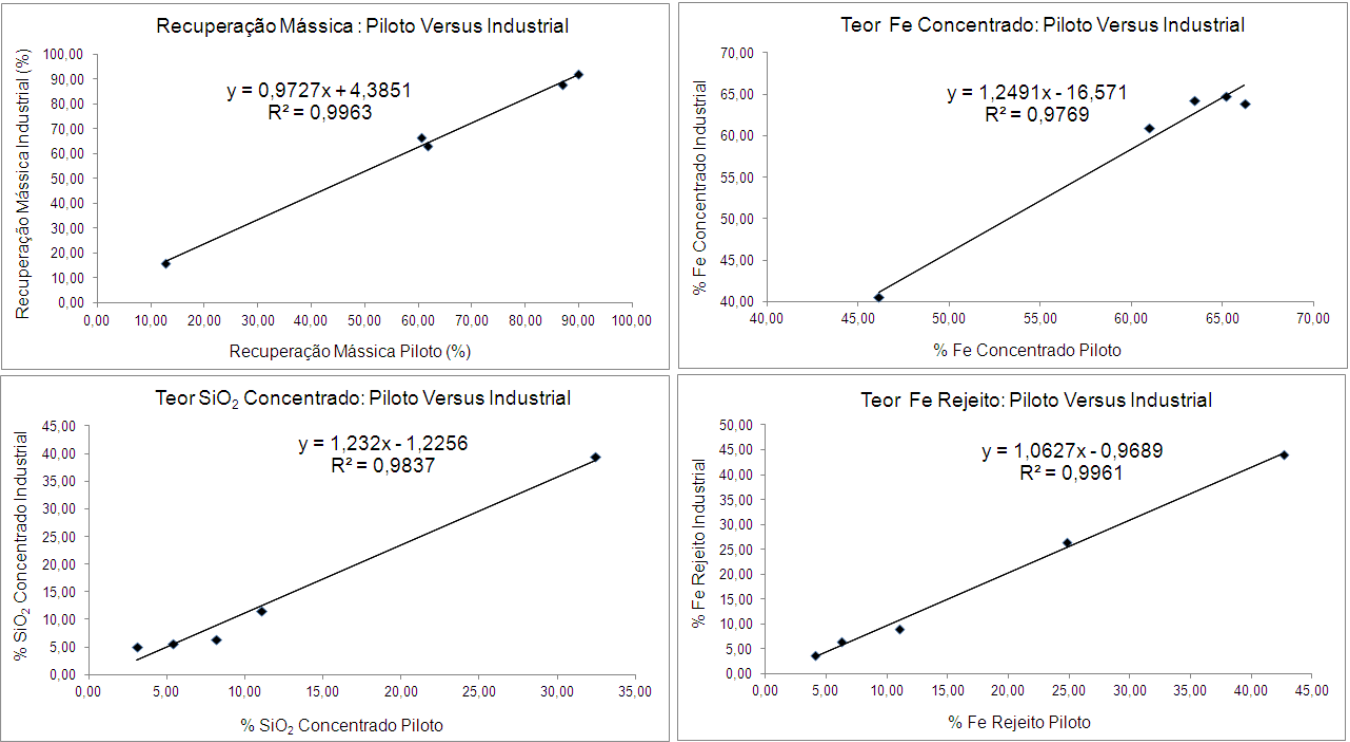

Figura 7 - Gráficos de correlação dos resultados piloto versus industrial.

* Contribuição técnica ao 44ํㅗㄴ Seminário de Redução de Minério de Ferro e Matérias-primas, 15ㅇ Simpósio Brasileiro de Minério de Ferro e 2o Simpósio Brasileiro de Aglomeração de Minério de Ferro, 15 a 18 de setembro de 2014, Belo Horizonte, MG, Brasil. 


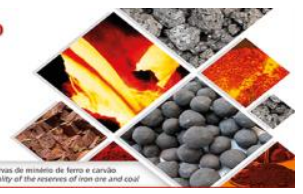

\subsection{Testes de Bancada}

A tabela 3 apresenta um comparativo dos resultados de bancada versus piloto. Dentre os vários testes de bancada executados na primeira batelada, utilizou-se aquele realizado na mesma condição de campo magnético da piloto. Vale lembrar que os testes de bancada foram realizados no concentrador eletromagnético estático modelo L4 e os testes piloto no concentrador eletromagnético contínuo Minimag (nome comercial). Para a maioria das demais amostras, os testes de bancada apresentam em relação a piloto, menores teores de $\mathrm{SiO}_{2}$ no concentrado e maiores teores de $\mathrm{Fe}$ no rejeito. Acredita-se que isso esteja associado à característica de cada amostra somada à limitação operacional que comumente os concentradores estáticos de bancada apresentam, uma vez que eles não possuem os mesmos mecanismos de atuação de um concentrador WHIMS de alimentação contínua (piloto/industrial). A figura 8 apresenta gráficos de correlação entre os testes de bancada versus piloto. Apesar do pequeno espaço amostral (apenas cinco pontos), verifica-se uma certa linha de tendência. Dessa forma, entende-se que é possível correlacionar os resultados obtidos nos testes bancada com os resultados da piloto. Nota-se que a correlação não é ótima, mas é boa, apresentando $R^{2}$ superior a 0,77 . Acredita-se que aumentando o espaço amostral, é possível fortalecer o nível dessas correlações e, assim poder utilizar as equações dos gráficos para transpor os resultados de bancada para piloto.

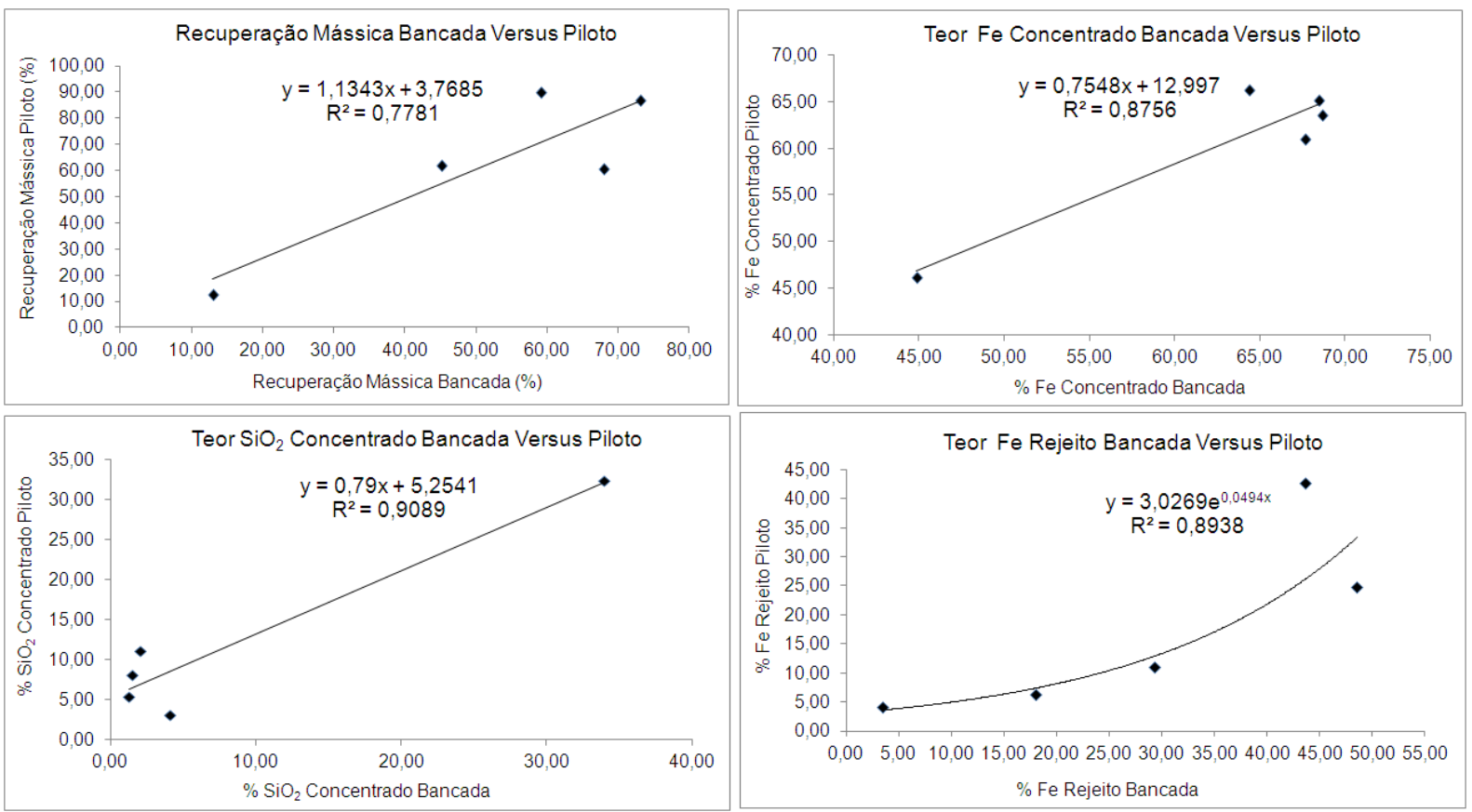

Figura 8: Gráficos de correlação dos resultados bancada versus piloto (1를 batelada de testes).

* Contribuição técnica ao 44 Seminário de Redução de Minério de Ferro e Matérias-primas, 15은 Simpósio Brasileiro de Minério de Ferro e 2ํ Simpósio Brasileiro de Aglomeração de Minério de Ferro, 15 a 18 de setembro de 2014, Belo Horizonte, MG, Brasil. 
Tabela 3: Resultados dos testes de bancada versus piloto (1를 batelada de testes).

\begin{tabular}{|c|c|c|c|c|c|c|c|c|c|c|}
\hline \multirow{2}{*}{ Amostra } & \multirow{2}{*}{ Escala } & \multirow{2}{*}{ Fluxo } & \multirow{2}{*}{$\begin{array}{c}\text { Recuperação } \\
\text { Mássica (\%) }\end{array}$} & \multicolumn{5}{|c|}{ Análise Química Global (\%) } & \multirow{2}{*}{$\begin{array}{l}\text { Campo } \\
\text { (Gauss) }\end{array}$} & \multirow{2}{*}{$\begin{array}{l}\text { Gap } \\
\text { (mm) }\end{array}$} \\
\hline & & & & $\mathrm{Fe}$ & $\mathrm{SiO}_{2}$ & $\mathrm{P}$ & $\mathrm{Al}_{2} \mathrm{O}_{3}$ & Mn & & \\
\hline \multirow{6}{*}{$\begin{array}{c}\text { Alimentação } \\
\text { Jones Finos } \\
\text { Cauê }\end{array}$} & \multirow{3}{*}{ Bancada } & Alimentação & 100,00 & 40,96 & 41,06 & 0,023 & 0,74 & 0,074 & \multirow{3}{*}{9.000} & \multirow{3}{*}{2,5} \\
\hline & & Concentrado & 45,18 & 67,66 & 2,06 & 0,020 & 0,37 & 0,077 & & \\
\hline & & Rejeito & 54,82 & 18,06 & 73,61 & 0,014 & 0,59 & 0,048 & & \\
\hline & \multirow{3}{*}{ Piloto } & Alimentação & 100,00 & 40,05 & 41,11 & 0,019 & 0,80 & 0,087 & \multirow{3}{*}{9.000} & \multirow{3}{*}{2,5} \\
\hline & & Concentrado & 61,75 & 60,96 & 11,10 & 0,019 & 0,52 & 0,079 & & \\
\hline & & Rejeito & 38,25 & 6,28 & 89,51 & 0,018 & 1,22 & 0,099 & & \\
\hline \multirow{6}{*}{$\begin{array}{c}\text { Alimentação } \\
\text { Jones } \\
\text { Alegria }\end{array}$} & \multirow{3}{*}{ Bancada } & Alimentação & 100,00 & 8,90 & 86,37 & 0,028 & 0,86 & 0,001 & \multirow{3}{*}{13.000} & \multirow{3}{*}{1,5} \\
\hline & & Concentrado & 12,98 & 44,86 & 34,01 & 0,018 & 0,89 & 0,015 & & \\
\hline & & Rejeito & 89,20 & 3,45 & 93,85 & 0,009 & 0,56 & 0,002 & & \\
\hline & \multirow{3}{*}{ Piloto } & Alimentação & 100,00 & 9,39 & 85,24 & 0,010 & 0,76 & 0,001 & \multirow{3}{*}{13.000} & \multirow{3}{*}{1,5} \\
\hline & & Concentrado & 12,65 & 46,16 & 32,47 & 0,015 & 0,85 & 0,003 & & \\
\hline & & Rejeito & 87,35 & 4,06 & 92,88 & 0,009 & 0,74 & 0,001 & & \\
\hline \multirow{6}{*}{$\begin{array}{c}\text { Alimentação } \\
\text { Jones } \\
\text { Rougher } \\
\text { Brucutu }\end{array}$} & \multirow{3}{*}{ Bancada } & Alimentação & 100,00 & 56,00 & 18,02 & 0,011 & 0,39 & 0,023 & \multirow{3}{*}{12.600} & \multirow{3}{*}{2,5} \\
\hline & & Concentrado & 73,16 & 68,65 & 1,54 & 0,014 & 0,26 & 0,016 & & \\
\hline & & Rejeito & 26,84 & 29,30 & 57,75 & 0,015 & 0,35 & 0,009 & & \\
\hline & \multirow{3}{*}{ Piloto } & Alimentação & 100,00 & 56,64 & 17,81 & 0,010 & 0,42 & 0,011 & \multirow{3}{*}{12.600} & \multirow{3}{*}{2,5} \\
\hline & & Concentrado & 86,96 & 63,49 & 8,17 & 0,009 & 0,37 & 0,010 & & \\
\hline & & Rejeito & 13,04 & 10,98 & 82,04 & 0,014 & 0,73 & 0,015 & & \\
\hline & & Alimentação & 100,00 & 60,68 & 11,78 & 0,019 & 0,41 & 0,019 & & \\
\hline Alimentacão & Bancada & Concentrado & 59,13 & 68,45 & 1,26 & 0,016 & 0,36 & 0,019 & 9.000 & 2,5 \\
\hline Jones & & Rejeito & 40,87 & 48,53 & 28,95 & 0,024 & 0,63 & 0,016 & & \\
\hline $\begin{array}{l}\text { Cleaner } \\
\text { Brucutu }\end{array}$ & & Alimentação & 100,00 & 61,28 & 10,76 & 0,017 & 0,54 & 0,009 & & \\
\hline & Piloto & Concentrado & 89,94 & 65,18 & 5,39 & 0,017 & 0,42 & 0,010 & 9.000 & 2,5 \\
\hline & & Rejeito & 10,06 & 24,79 & 61,44 & 0,027 & 1,12 & 0,013 & & \\
\hline & & Alimentação & 100,00 & 58,28 & 14,37 & 0,053 & 0,96 & 0,250 & & \\
\hline & Bancada & Concentrado & 67,97 & 64,40 & 4,10 & 0,048 & 0,93 & 0,100 & 12.000 & 3,8 \\
\hline Jones & & Rejeito & 32,03 & 43,60 & 35,20 & 0,047 & 0,97 & 0,342 & & \\
\hline Rougher & & Alimentação & 100,00 & 56,98 & 15,14 & 0,044 & 0,85 & 0,275 & & \\
\hline & Piloto & Concentrado & 60,67 & 66,23 & 3,08 & 0,033 & 0,42 & 0,126 & 12.000 & 3,8 \\
\hline & & Rejeito & 39,33 & 42,68 & 33,83 & 0,064 & 1,67 & 0,563 & & \\
\hline
\end{tabular}

Visando aumentar o espaço amostral e confirmar os resultados de correlação obtidos entre os testes de bancada e piloto, realizou-se uma segunda batelada de testes. Para tanto, utilizou-se cinco amostras oriundas da Barragem de Fábrica (unidade Vale, localizada às margens da BR 040 à aproximadamente $70 \mathrm{~km}$ de Belo Horizonte). De maneira geral verificou-se para essa segunda batelada de testes a mesma tendência da primeira, mantendo-se assim o nível de correlação. A figura 9 apresenta gráficos de correlação entre os testes de bancada versus piloto, acumulando os resultados da primeira e segunda batelada de testes. Apesar do espaço amostral ainda ser pequeno (dez pontos), verifica-se que ao inserir os resultados da segunda batelada de testes, a tendência e o nível de correlação continuaram os mesmos, o que ratifica a possibilidade de correlacionar os testes bancada com os testes piloto, utilizando as equações dos gráficos para transpor os resultados. Isso traria um ganho muito enorme para os trabalhos de caracterização envolvendo o processo de concentração magnética, uma vez que, atualmente no CPT, é necessário uma massa de amostra de furo de sonda de aproximadamente $80 \mathrm{~kg}$, para viabilizar a realização de um teste piloto de concentração magnética. Em caso de realização de somente testes de bancada, essa massa reduziria para $20 \mathrm{~kg}$, ou seja, quatro vezes menos. Uma redução significativa, que proporcionaria ganhos

* Contribuição técnica ao 44 Seminário de Redução de Minério de Ferro e Matérias-primas, 15ㅇ Simpósio Brasileiro de Minério de Ferro e 2o Simpósio Brasileiro de Aglomeração de Minério de Ferro, 15 a 18 de setembro de 2014, Belo Horizonte, MG, Brasil. 
tangíveis como: redução de custos (amostra de furo de sonda possui custo elevado) e redução de tempo (testes piloto por serem mais complexos, são também mais morosos).

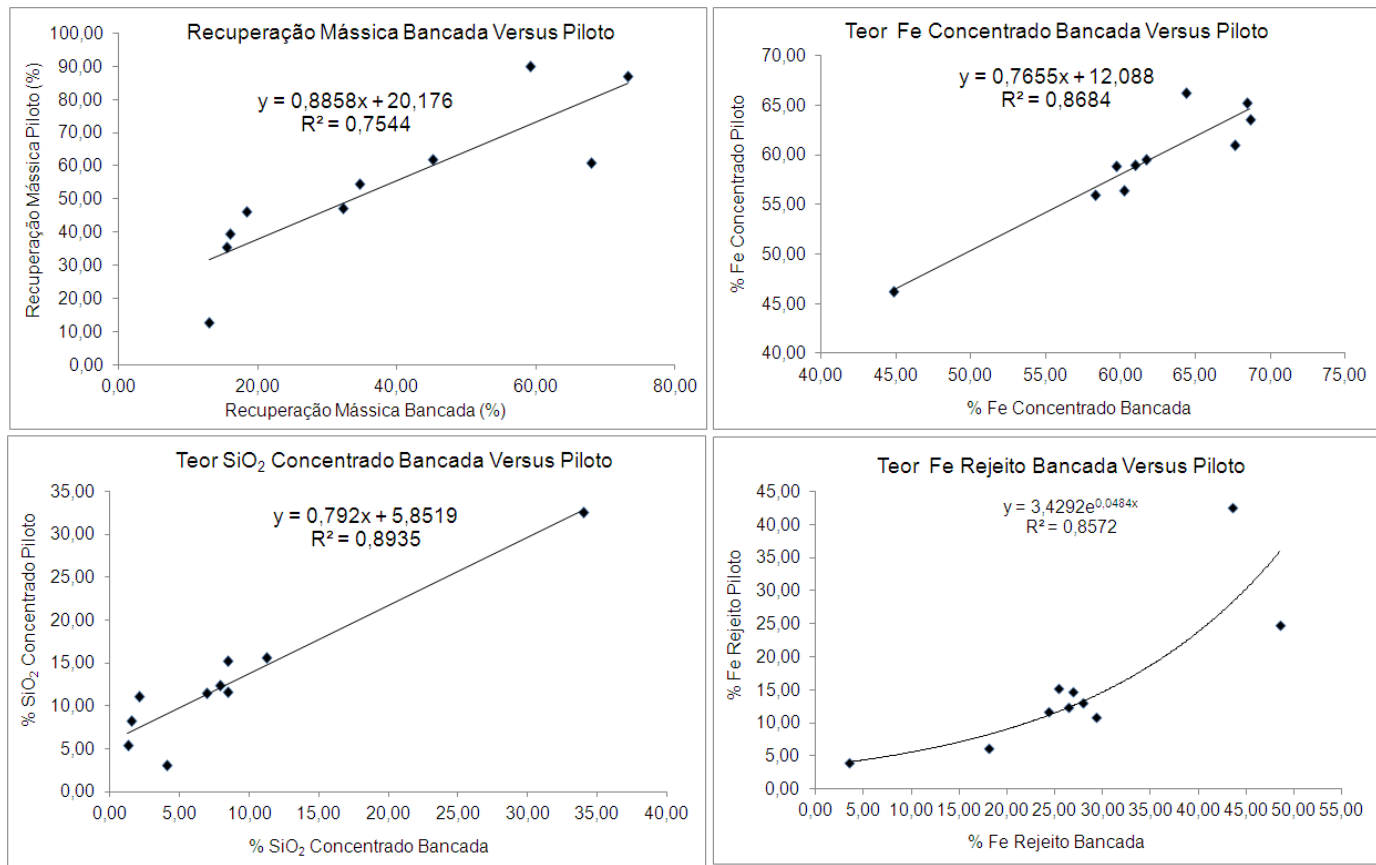

Figura 9: Gráficos de correlação dos resultados bancada versus piloto (acumulado $1^{\underline{a}}$ e $2^{\underline{a}}$ batelada de testes.

Utilizando as equações dos gráficos de correlações (figura 9), calculou-se para as amostras da segunda batelada de testes, os teores de $\mathrm{Fe}$ e $\mathrm{SiO}_{2}$ do concentrado e o teor de $\mathrm{Fe}$ do rejeito. Por apresentar $\mathrm{R}^{2}$ mais baixo (correlação mais fraca), a recuperação mássica não foi contemplada. A tabela 4 apresenta um comparativo entre os resultados "Piloto Real" (obtidos nos testes) e "Piloto Calculado" (calculado pelas equações dos gráficos de correlação da figura 10). Nota-se que apesar de alguns desvios, os resultados calculados são similares aos resultados reais. Isso confirma que realmente é possível utilizar equações de correlação para estimar resultados piloto a partir dos resultados de bancada. Entretanto, entende-se que para este o trabalho, isso ainda não seria possível, uma vez que, o espaço amostral (número de amostras estudadas) é pequeno.

Tabela 4 - Comparativo dos resultados piloto real versus piloto calculado.

\begin{tabular}{c|c||cc||c|c}
\hline \multicolumn{2}{c||}{ Teor Fe Concentrado } & \multicolumn{2}{c|}{ Teor $\mathrm{SiO}_{2}$ Concentrado } & \multicolumn{2}{c}{ Teor Fe Rejeito } \\
\cline { 3 - 6 } \cline { 4 - 6 } Piloto Real & Piloto Calculado & Piloto Real & Piloto Calculado & Piloto Real & Piloto Calculado \\
\cline { 3 - 6 } & 59,48 & 11,52 & 11,32 & 12,49 & 12,29 \\
56,34 & 58,17 & 15,22 & 12,58 & 11,80 & 11,15 \\
55,98 & 56,72 & 15,61 & 14,74 & 15,26 & 11,71 \\
58,91 & 58,77 & 12,37 & 12,10 & 13,09 & 13,28 \\
58,87 & 57,82 & 11,64 & 12,57 & 14,76 & 12,61 \\
\hline
\end{tabular}

\section{CONCLUSÕES}

- Este trabalho confirma que a interpretação mineralógica é uma boa ferramenta para estimar recuperação mássica e qualidade de concentrado

* Contribuição técnica ao 44ํㅗㄴ Seminário de Redução de Minério de Ferro e Matérias-primas, 15ㅇ Simpósio Brasileiro de Minério de Ferro e 2o Simpósio Brasileiro de Aglomeração de Minério de Ferro, 15 a 18 de setembro de 2014, Belo Horizonte, MG, Brasil. 


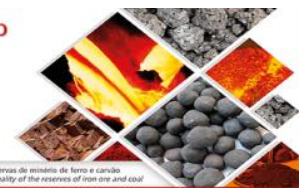

para os processos de concentração magnética, o que possibilita ter 0 conhecimento prévio sobre o potencial de concentrabilidade de uma determinada amostra.

- A interpretação mineralógica realizada coma amostra global apresentou correlações mais fortes que a amostra fracionada, sugerindo maior previsibilidade do modelo utilizado atualmente pelo CPT.

- Os resultados dos testes piloto ficaram praticamente idênticos aos resultados da industrial, apresentando correlações fortes com $R^{2}$ acima de 0,97. Assim, entende-se que não é necessário aplicar nenhum fator de conversão dos resultados piloto para a industrial. Eles podem ser utilizados diretamente nos projetos de minério de ferro, no que tange a configuração e dimensionamento de circuitos de concentração magnética

- Este trabalho confirma que é possível correlacionar os resultados obtidos nos testes bancada com os resultados piloto. Os gráficos de correlação apresentaram $\mathrm{R}^{2}$ superior a 0,77 (não são corelações ótimas, mas são boas). Assim, verifica-se que é possível utilizar equações de correlação para estimar resultados piloto, a partir dos resultados de bancada. Entretanto, entende-se que para este o trabalho, isso ainda não seria possível, uma vez que, o espaço amostral (número de amostras estudadas) é pequeno.

- Sugere-se estudar interpretação mineralógica para amostras fracionadas em um número maior de faixas. Acredita-se que o resultado negativo com amostra fracionada neste trabalho, esteja associado ao pequeno número de faixas (somente duas: $<0,045 \mathrm{~mm}$ e $<0,045 \mathrm{~mm}$ ).

- Visando aumentar o espaço amostral e, consequentemente melhorar a força das correlações, sugere-se a realização de testes adicionais de concentração magnética nas escalas de bancada e piloto. Separar esses testes por abertura do gap e granulometria de alimentação. Acredita-se que as correlações serão mais fortes em grupos de testes/amostras mais homogêneos. A realização de um maior número de testes tornará essa separação possível.

\section{Agradecimentos}

Os autores agradecem à CAPES/PROEX, CNPq e FAPEMIG pelo apoio financeiro ao PPGEM e à VALE pelo financiamento do projeto.

\section{REFERÊNCIAS}

1 Klein C, Dutrow B. Crystal growth and defects; twinning, color, and magnetism. In: Klein C, Dutrow B. The Manual of Mineral Science. 23. ed. John Wiley \& Sons, INC, 2007a, Chapter 10, p.217-244.

2 Klein C, Dutrow B. Physical properties of minerals. In: KLEIN, C. and DUTROW B. The Manual of Mineral Science. 23. ed. John Wiley \& Sons, INC, 2007b, Chapter 2, p.19-36.

3 Wasmuth HD, Unkelbach KH. Recent Developments in Magnetic Separation of Feebly Magnetic Minerals. Minerals Engineering, 1991; 4: 825-837.

4 Paula NN. Treinamento de Concentração Magnética Convencional Aplicada em Minério de Ferro. Belo Horizonte: Vale; 2009.

* Contribuição técnica ao 44 Seminário de Redução de Minério de Ferro e Matérias-primas, $15^{\circ}$ Simpósio Brasileiro de Minério de Ferro e 2ํ Simpósio Brasileiro de Aglomeração de Minério de Ferro, 15 a 18 de setembro de 2014, Belo Horizonte, MG, Brasil. 